\title{
An arbitration algorithm for multiport memory systems
}

\author{
Alex A. Aravind ${ }^{a)}$ \\ Department of Computer Science, \\ University of Northern British Columbia, \\ Prince George, British Columbia, Canada \\ a) csalex@unbc.ca
}

Abstract: Multiport memories are increasingly used in smart-phones, multimode handsets, multiprocessor systems, network processors, graphics chips, and other high performance electronic devices $[1,2,4,8]$. This paper presents a fully distributed software solution to the arbitration problem in multiport memory systems. Our solution is simple, efficient, and assures $L R U$ fairness.

Keywords: multiport memory, arbitration, shared memory

Classification: Storage technology

\section{References}

[1] C. Springer, "Enabling Multimode Handsets," EE Times, Oct. 2004.

[2] L. E. Frenzel, "Dual-Port SRAM Accelerates Smart-Phone Development," Electronic Design, Feb. 2004.

[3] G. Taubenfeld, "The Black-White Bakery Algorithm and Related Bounded-Space, Adaptive, Local-Spinning and FIFO Algorithms," Lecture Notes in Computer Science, vol. 3274, pp. 56-70, 2004.

[4] C.-W. Wang, K.-L. Cheng, C.-T. Huang, and C.-W. Wu, "Test and Diagnosis of Word-Oriented Multiport Memories," Proc. of the 21st IEEE VLSI Test Symposium, pp. 248-253, 2003.

[5] J. H. Anderson, Y.-J. Kim, and T. Herman, "Shared-memory Mutual Exclusion: Major Research Trends Since 1986," Distributed Computing, no. 16, pp. $75-110,2003$.

[6] J. H. Anderson and Y.-J. Kim, "Nonatomic Mutual Exclusion with Local Spinning," Proc. of the ACM Sym. on PODC, pp. 3-12, 2002.

[7] T. Raineault, "Semaphores Aid Multiprocessor Designs," Embedded Edge, pp. 14-20, Oct. 2001.

[8] R. Stodieck, "The IDT FourPort SRAM Facilitates Multiprocessor Design," IDT Application Note An-43, March 2000.

[9] M. Raynal, Algorithms for Mutual Exclusion Problem, The MIT Press, Cambridge, Massachusetts, 1986.

[10] L. Lamport, "The Mutual Exclusion Problem Part II: Statement and Solutions," J. ACM, vol. 33, no. 2, pp. 323-326, 1986.

[11] L. Lamport, "A New Solution of Dijkstra's Concurrent Programming Problem," Comm. ACM, vol. 17, no. 8, pp. 453-455, 1974. 


\section{Introduction}

Multiport memories are increasingly used in smart-phones, multimode handsets, multiprocessor systems, network processors, graphics chips, and other high performance electronic devices $[1,2,4,8]$. Multiport memory allows concurrent accesses to memory words through multiple ports.

Resolving conflicting accesses to shared memory by concurrent processors, also called memory arbitration or mutual exclusion, is a fundamental problem in concurrent computing. Distributed solution to this problem is generally complex and many arbitration algorithms are available for single port memory systems $[9,5]$. The problem becomes more complex for multiport memory systems, due to the possibility of concurrent accesses to individual memory words through many ports. Among the solutions proposed for single port memory systems, the algorithms presented in $[11,10,6]$ can be used to solve the arbitration problem in multiport memory systems. We briefly review them here.

Lamport's Bakery algorithm presented in [11] is the simplest and popularly known arbitration algorithm. It is based on the idea of using token numbers to resolve the conflict among the competing processors. This algorithm has a practical limitation that the token numbers can grow unboundedly, if always some processor is in need of using the shared memory. There are many recent attempts to bound the token values [3] and all these attempts require exclusive access to the shared variables (memory words). Therefore, they cannot be used in multiport memory systems. Another algorithm, by Lamport, with many nice properties is incrementally developed and presented in [10]. However, this algorithm is conceptually difficult for an average designer to implement. Recently, an algorithm for distributed shared memory systems - where the memory access is non-uniform - is presented in [6]. Also, after mentioning hardware based solutions such as hardware interrupt masking, hardware semaphores, and stalling processors with busy logic, a software based Master/Slave control protocol for a multiport memory systems is presented in [8].

Among the various criteria of the arbitration algorithms, the fairness property is very crucial one. It decides which processor among the competing processors is allowed to succeed next. First In First Out (FIFO) and Least Recently Used $(L R U)$ are two important fairness criteria widely used in many applications. $L R U$ favors the infrequent users of the resource compared to the frequent users. There are many algorithms available in the literature $[9,5]$ to assure FIFO. To the best of our knowledge, no arbitration algorithm is presented for shared memory system with $L R U$ fairness criterion.

This paper presents a fully distributed software solution to the arbitration problem in multiport memory systems. Our algorithm is simple, assures $L R U$ fairness, and applicable for multiport memory systems. 


\section{System Model and Problem Statement}

We consider a multiport shared memory system of $n$-processors with ids $1,2, \ldots, n$. The processors can simultaneously access the same memory location, may be through independent ports. The execution speed of any processor is finite but unpredictable.

We assume $R$ as the memory segment that requires mutually exclusive access among the processors. The memory arbitration problem is to design an algorithm that assures the following properties: (i) at any time, at most one processor is allowed to access $R$ (safety property) and (ii) when one or more processors interested in accessing $R$, one of them eventually succeeds in accessing $R$ (liveness property). In addition to these two properties the following is a desirable property: (iii) any processor interested in accessing $R$ will be able to do so in finite time (freedom from starvation property). We also assume that a processor will neither accesses $R$ continously forever nor fails when it is accessing $R$.

The code segment that a competing processor executes can be divided into two parts: the part which accesses the shared memory $R$ (Critical Section $(C S))$ and the remaining part (Noncritical Section (NCS)). A solution to the arbitration problem has essentially two components:Entry Section and Exit Section. These components has to be designed and inserted appropriately in the codes that all the competing processors execute to ensure consistent access to $R$.

\section{The Least Recently Used ( $L R U)$ Algorithm}

\subsection{Idea}

The basic idea behind the algorithm is very simple that, among the competing processors, the processor who accessed the CS "least recently" succeeds to access the CS next. The popular way to implement this idea is by using the timestamp (clock value) of each processor's latest access to the CS. Unfortunately, this approach requires unbounded size shared variables, similar to token variables in bakery algorithm, to hold the timestamp values. In this paper, we introduce a different approach to implement the $L R U$ idea. Instead of timestamps, our algorithm uses the order of most recent CS accesses of the processors to choose the least recently CS accessed processor. The appeal of our approach is that it uses only bounded size shared variables and works for multiport memory systems.

\subsection{Algorithm Design}

We use an integer array called pos of size $n$ to hold relative positions of the recent CS accesses (we refer as $L R U$ positions) of the processors. The cell pos[1] holds the $i d$ of "least recently" CS accessed processor, pos[2] holds the $i d$ of next least recently CS accessed processor, etc., and $p o s[n]$ holds the $i d$ of "most recently" CS accessed processor. That is, between any two processors $p$ and $q$ with respective $L R U$ position values $i$ and $j$, if $i>j$ then the processor $q$ has higher priority than $p$ in accessing the CS. Each 
processor uses a local variable $k$ to keep track of its $L R U$ position in pos. A boolean array competing of size $n$ is used to indicate processors' interest in accessing the CS. Initially, for each $p, \operatorname{pos}[p]$ is set to $p$, competing $[p]$ is set to false, and $k$ is set to $n$. If $k$ is not the current $L R U$ position of a processor $p$, then it can find it by scanning the pos array downwards from location $k$ as follows:

while $(\operatorname{pos}[k] \neq p) k:=k-1$;

In the entry section, a filter mechanism is used to block the lower priority processors. A blocked processor, say $p$, can cross the filter only after all the higher priority processors complete their CS executions. The filter essentially has two components: (i) checking for the competition of higher priority processors and (ii) waiting for the higher priority processors to complete their CS accesses and leave the competition.

If there exists a higher priority processor $q$ with $L R U$ position less than $k$ is competing for the CS, then $p$ waits until $q$ completes the CS access and leaves the competition. Since each higher priority processor changes the $L R U$ positions of all the lower priority processors before it exits, a lower priority processor $p$ can simply wait for its position change before it checking for any other higher priority processors. Thus, the filter for a processor $p$ is designed as follows:

while $(\exists j,(1 \leq j<k) \wedge($ competing $[\operatorname{pos}[j]]=$ true $))$

$\{$ wait until $(\operatorname{pos}[k] \neq p)$; while $(\operatorname{pos}[k] \neq p) k:=k-1 ;\}$

Next, we design a safety-net to assure exclusive access to the CS. Suppose a processor $p$ starts its competition, determines that no higher priority processor is competing, and therefore proceeds further to enter the CS. Now, a higher priority processor $q$ starts its competition and determines a lower priority processor $p$ is competing. From $q$ 's point of view, $p$ could be either blocked in the filter or in the CS (that is outside the filter). To avoid this dilemma, we introduce a boolean array called in_cs of size $n$ to indicate whether the processors crossed the filter or not. The safety net will allow a processor to cross it only when in_cs of all other processors are false. (in_cs $[p]$ is initialized to false, for all $p$ ). The safety-net for $p$ is given next. repeat \{

in_cs $[p]:=$ false; filter; in_cs $[p]:=$ true;

\}until $(\forall j \neq p$, in_cs $[j]=$ false $)$

Finally, after completing the CS access, the processor $p$ adjusts the $L R U$ positions as follows:

while $(\operatorname{pos}[k] \neq p) k:=k-1$;

$\operatorname{for}(j:=k$ to $n-1) \operatorname{pos}[j]:=\operatorname{pos}[j+1]$;

$\operatorname{pos}[n]:=p$;

The $L R U$ positions adjustment basically promotes the processors between location $k+1$ and $n$ by shifting one position left and places the id of $p$ at location $n$. Since each position adjustment (by (c)) is done exclusively after every CS access completion, it is easy to see that it preserves the ids of all processors in pos. Also, it is obvious that a processor can find its id (by (a)) during no $L R U$ position shift. Suppose $p$ is reading at $p o s[j]$ while $q$ is writing 
the value of $\operatorname{pos}[j+1]$ on $\operatorname{pos}[j]$ for a position shift, then it is easy to see that $\operatorname{pos}[j+1]$ cannot be equal to $p$ (otherwise, since $p$ is scanning from right to left, it must have find its id at $\operatorname{pos}[j+1]$ and therefore blocked at $\operatorname{pos}[j+1]$ (in the filter). If the value of $\operatorname{pos}[j]$ is $p$, before $q$ writes on it, then the current value of $\operatorname{pos}[j-1]$ must be $p$ (due to shift). Therefore, even if $p$ reads $\operatorname{pos}[j]$ value as $p$ during a shift and blocks at $\operatorname{pos}[j]$, it will eventually read the correct value of $p o s[j]$ and proceed further to identify its $L R U$ position. That is, the possibility of inconsistent read due to multiport memory will not affect the correct reading of the $L R U$ positions. The complete algorithm for the processor $p$ is presented in Figure 1.

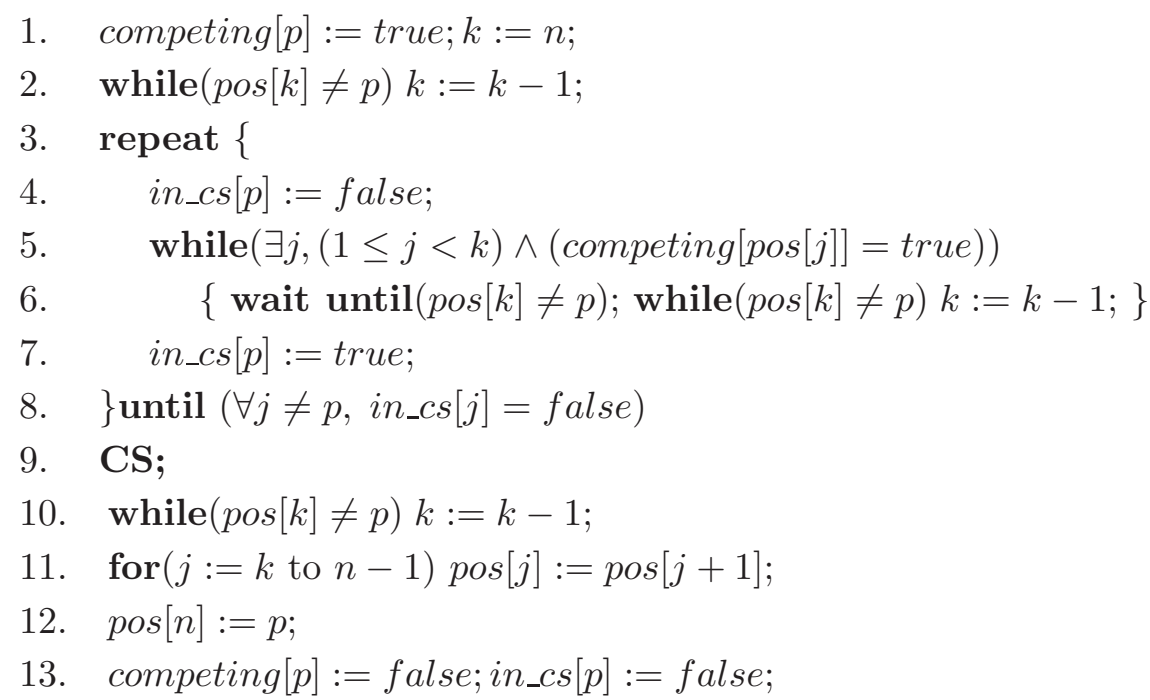

Fig. 1. $L R U$ Algorithm

\section{Correctness Proofs}

First we introduce some terminology. We denote: (i) $r_{i}(x)$ and $w_{i}(x)$, respectively, as the read and write operations of processor $i$ on the variable $x$; (ii) an event $e_{i}$ occurred before another event $e_{j}$ as $e_{i} \rightarrow e_{j}$. We say $e_{i} \rightarrow e_{j}$ as $e_{i}$ precedes $e_{j}$ in time; (iii) an event $e_{i}$ does not occurred before another event $e_{j}$ as $e_{i} \not e_{j}$; (iv) occurrences of two events $e_{1}$ and $e_{2}$ that overlap in time as $e_{1} \| e_{2}$; and (v) occurrences of two events $e_{1}$ and $e_{2}$ that do not overlap in time as $e_{1} \backslash \mid e_{2}$.

Theorem 4.1 The LRU algorithm assures mutual exclusion.

Proof: Proof by contradiction. Suppose there are more than one processors simultaneously accessing the CS. In particular, we consider two such processors $i$ and $j$ that access the CS at same time. Each of these two processors must have observed the other's in_cs value as false, to cross the line 8 before entering the CS. Let $w_{i}\left(i n_{-} c s[i]\right)$ and $r_{i}\left(i n_{-} c s[j]\right)$, respectively, be the latest write on $i n_{-} c s[i]$ at line 7 and the latest read on $i n_{-} c s[j]$ at line 8 by the processor $i$ before it entered the CS. Similarly, let $w_{j}\left(i n_{-} c s[j]\right)$ and $r_{j}\left(i n_{-} c s[i]\right)$, 
respectively, be the latest write on $i n_{-} c s[j]$ at line 7 and the latest read on $i n_{-} c s[i]$ at line 8 by the processor $j$ before it entered the CS. The read and write operations of same processor cannot overlap in time. Therefore,

$w_{i}\left(i n_{-} c s[i]\right) \rightarrow r_{i}\left(i_{-} \_s s[j]\right)$

and

$w_{j}\left(i n_{-} c s[j]\right) \rightarrow r_{j}\left(i n_{-} c s[i]\right)$

If $w_{j}\left(i n_{-} c s[j]\right) \rightarrow r_{i}\left(i n_{-} c s[j]\right)$ then the processor $i$ must have read the value of $i n_{-} c s[j]$ as true at line 8 before entering the CS, which is a contradiction. Similarly, if $w_{i}\left(i_{-} \_s[i]\right) \rightarrow r_{j}\left(i n_{-} c s[i]\right)$ then the processor $j$ must have read the value of $i n_{-} c s[i]$ as true at line 8 before entering the CS, which is also a contradiction.

Without loss of generality, assume that $w_{j}\left(i n_{-} c s[j]\right) / r_{i}\left(i n_{-} c s[j]\right)$ It is enough to prove that $w_{i}\left(i n_{-} c s[i]\right) \rightarrow r_{j}\left(i n_{-} c s[i]\right)$. Relation (3) implies either $r_{i}\left(i n_{-} c s[j]\right) \rightarrow w_{j}\left(i n_{-} c s[j]\right)$ or $r_{i}\left(i n_{-} c s[j]\right) \| w_{j}\left(i n_{-} c s[j]\right)$. If $r_{i}\left(i n_{-} c s[j]\right) \rightarrow$ $w_{j}\left(i n_{-} c s[j]\right)$, then by $(1)$ and $(2)$, we get $w_{i}\left(i n_{-} c s[i]\right) \rightarrow r_{j}\left(i n_{-} c s[i]\right)$. If $r_{i}\left(i n_{-} c s[j]\right) \| w_{j}\left(i n_{-} c s[j]\right)$, then by (1), $w_{j}\left(i n_{-} c s[j]\right) \nrightarrow w_{i}\left(i n_{-} c s[i]\right)$. This means either $w_{i}\left(i n_{-} c s[i]\right) \rightarrow w_{j}\left(i n_{-} c s[j]\right)$ or $w_{j}\left(i n_{-} c s[j]\right) \| w_{i}\left(i n_{-} c s[i]\right)$.

$$
\begin{aligned}
\text { Case 1: } & w_{i}(\text { in_cs }[i]) \rightarrow w_{j}\left(i n_{-} c s[j]\right) \\
& \Rightarrow w_{i}(\text { in_cs }[i]) \rightarrow w_{j}(\text { in_cs }[j]) \rightarrow r_{j}\left(i n_{-} c s[i]\right) \\
& \Rightarrow w_{i}\left(i n_{-} c s[i]\right) \rightarrow r_{j}\left(i n_{-} c s[i]\right)
\end{aligned}
$$

Case 2: $w_{i}\left(i n_{-} \_s[i]\right) \| w_{j}\left(i n_{-} c s[j]\right)$

From the hypothesis, we get $w_{j}\left(i_{-} \_s[j]\right)$ overlaps with both $w_{i}\left(i n_{-} c s[i]\right)$ and $r_{i}\left(i n_{-} c s[j]\right)$. By (1) $w_{i}\left(i n_{-} c s[i]\right)$ must terminate before $w_{j}\left(i n_{-} c s[j]\right)$ terminates, for $r_{i}\left(i n_{-} c s[j]\right)$ to overlap with $w_{j}\left(i n_{-} c s[j]\right)$. This implies, and by (2), $w_{i}($ in_cs $[i])$ must terminate before $r_{j}\left(i n_{-} c s[i]\right)$ starts. That is, $w_{i}\left(i n_{-} c s[i]\right) \rightarrow$ $r_{j}\left(i n_{-} c s[i]\right)$. Hence the proof.

\section{Theorem 4.2 The LRU algorithm assures liveness.}

Proof : If there is only one processor trying for its CS execution, then it can complete the lines 1 to 8 without blocking, and hence it can enter the $\mathrm{CS}$ in a finite time. If more than one processors are competing for the CS, then except the processor with the lowest $L R U$ position, all other processors will be blocked at the filter in lines 5 and 6 . This will eventually allow the processor with the lowest $L R U$ position to cross the line 8 and enter the CS.

Theorem 4.3 In LRU algorithm, the maximum number of overtakes possible over a processor to access the CS is $n-1$.

Proof : A processor after completing its CS execution, shifts the $L R U$ positions in lines 11 and 12 in such a way that it gets the lowest priority for the next turn. So, a processor cannot execute its CS consecutively when there are other processors currently competing for their CS executions. But, it is possible that a later processor can overtake an earlier arrived processor once due its current $L R U$ position. Since the maximum number of processors in the system is $n$, the maximum number of overtakes possible over a processor is $n-1$. 


\section{Conclusion}

In this paper, after briefly reviewing the hardware and software solutions for the arbitration problem in multiport memory systems, we presented a new algorithm. Our algorithm is simple, efficient, and assures $L R U$ fairness. 